$\mathrm{O}-\mathrm{\square}$

\title{
Surface Etching and Passivation of Black Phosphorus by Thermal Annealing
}

\author{
Sena Yang, ${ }^{1}$ Jeong Won Kim, ${ }^{1,2, *}$ \\ ${ }^{1}$ Korea Research Institute of Standards and Science (KRISS), 267 Gajeong-ro, Daejeon 34113, Korea \\ ${ }^{2}$ University of Science and Technology, 217 Gajeong-ro, Daejeon 34113, Korea \\ *corresponding author's e-mail: jeongwonk@kriss.re.kr
}

(Received: June 13, 2019; Accepted: June 18, 2019)

\begin{abstract}
Recently, next-generation two-dimensional (2D) materials to replace graphene inspired people to study other types of elements. Among them, black phosphorus (BP) have attracted great interests for their wide range of optical bandgap from near IR to visible wavelength and remarkable high hole mobility. However, one of critical challenges for the applications of BP to devices is its instability. Herein, we introduce a very simple method to fabricate a thin and stable BP. Thermal annealing in air above $200{ }^{\circ} \mathrm{C}$ creates a stable BP oxide layer on top of the BP surface. In the meantime, thermal oxidation reduces the thickness of the BP by etching the top BP layer. In addition, we find that the protective layer is composed of $\mathrm{P}_{2} \mathrm{O}_{5}$ by using $\mathrm{x}$-ray photoelectron spectroscopy (XPS). We suggest that the thermal annealing provides an effective and simple strategy to fabricate high-quality BP-based electronic devices.
\end{abstract}

\section{Introduction}

Since the discovery of two dimensional (2D) graphene, it has attracted attention as a next-generation electronic device material because of its semiconductor characteristics, excellent electrical and mechanical properties. However, the zero bandgap and chemical inertness of graphene limit its further application [1]. Therefore, in recent years, people have been inspired to study next-generation 2D materials beyond graphene. Among them, black phosphorus (BP) has emerged as an alternative 2D semiconductor material because its excellent electrical properties and variable bandgap with thickness like other transition metal dichalcogenides [2]. However, one of critical challenges to be overcome for wide applications to various devices is its chemical instability when it is exposed to water and air.

Here, we suggest a very simple and effective method to fabricate a thin and stable BP layer. A simple annealing treatment above $200{ }^{\circ} \mathrm{C}$ in ambient conditions creates a protective layer comprising phosphorous oxides on top of BP. In the meantime, thermal oxidation of the exposed BP and etching of the intrinsic BP layer reduces the $\mathrm{BP}$ thickness as observed by atomic force microscopy (AFM) measurements. In addition, we find that the protective layer on top of BP is composed of $\mathrm{P}_{2} \mathrm{O}_{5}$ species by using $\mathrm{x}$-ray photoelectron spectroscopy (XPS).

\section{Experimental}

\subsection{Sample fabrication by thermal annealing}

We fabricated BP flakes by mechanical exfoliation on a $\mathrm{SiO}_{2}(285 \mathrm{~nm}) / \mathrm{Si}(100)$ substrate using a Scotch tape. Thermal annealing processes were carried out on a hot plate in an ambient conditions above at $250{ }^{\circ} \mathrm{C}$.

\subsection{Characterization}

We obtained AFM images of BP flakes by using non-contact mode AFM of XE-70 (Park Systems Inc.). $\mathrm{X}$-ray photoelectron spectroscopy measurements on the BP were carried out using a Versaprobe II (Ulvac-Phi Inc). A monochromatized Al $\mathrm{K} \alpha$ source $(\mathrm{hv}=1486.6 \mathrm{eV})$ and a hemispherical electron analyzer provided a total energy resolution better than $0.5 \mathrm{eV}$.

\section{Results}

We exfoliate BP flakes on a $\mathrm{SiO}_{2} / \mathrm{Si}$ substrate in mechanical way and conducted AFM. Fig. 1(a) shows 

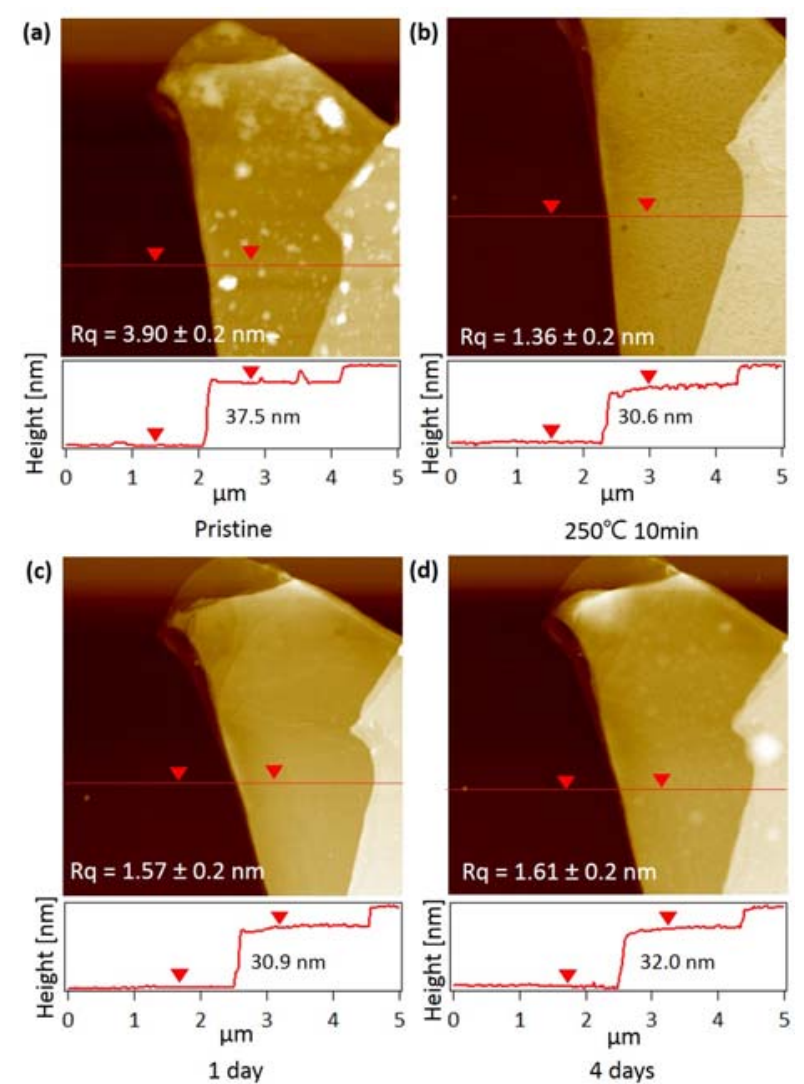

Fig. 1 AFM images and line profiles of BP flakes. (a) the pristine BP, (b) after annealing at $250{ }^{\circ} \mathrm{C}$ in air for $10 \mathrm{~min},(\mathrm{c})$ exposure to air for 1 day and (d) 4 days.

initial thickness of $37.5 \mathrm{~nm}$ and root mean square roughness $\left(R_{q}\right)$ of $3.90 \pm 0.2 \mathrm{~nm}$. Small bubbles on BP surface appear clearly in good agreement with previous study [3]. Thermal annealing of the BP flake at $250{ }^{\circ} \mathrm{C}$ for $10 \mathrm{~min}$ in an ambient condition decreases the BP thickness to $30.6 \mathrm{~nm}$ and $\mathrm{R}_{\mathrm{q}}$ to $1.36 \pm 0.2 \mathrm{~nm}$ as shown in Fig. 1(b). To verify improved stability of BP flake, AFM images with time after the annealing are compared. After one and four days of air exposure, the annealed BP flake exhibits still highly flat surface without any serious roughness change shown in Fig. 1 (c) and (d), Rq to $1.57 \pm 0.2 \mathrm{~nm}$ and $1.61 \pm 0.2 \mathrm{~nm}$, respectively. Even though a few bubbles evolve in four days, the overall surface morphology is not deteriorated. On the other hand, if BP without thermal annealing is exposed to air, a lot of bubbles are formed in a short time [4]. The above result indicates that the thermal annealing stabilizes BP layer in air condition. By the simple thermal method, we could fabricate stable and flat BP layer and decrease BP thickness.

XPS is performed to determine what kind of species is formed during the thermal annealing in air. XPS spectra are obtained before and after thermal annealing
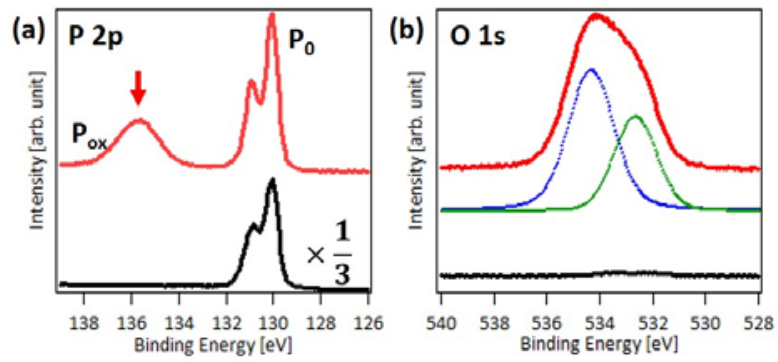

Fig. 2 (a) P 2p and (b) O 1s core-level XPS spectra of BP before (black) and after (red) thermal annealing.

for 60 min at $250{ }^{\circ} \mathrm{C}$ in ambient condition as shown in Fig. 2. In P 2p spectrum, the bare BP (black line) shows $\mathrm{P} 2 \mathrm{p}$ doublet of $\mathrm{P} 2 \mathrm{p}_{3 / 2}$ and $2 \mathrm{p}_{1 / 2}$ at 130.04 and 130.88 $\mathrm{eV}$, respectively. It is attributed to $\mathrm{P}_{0}$ corresponding to unreacted BP. After thermal annealing, a new broad peak appears at $135.41 \mathrm{eV}$ and $\mathrm{P}_{0}$ intensity significantly decreases as shown in red spectrum of Fig. 2(a). The new peak is assigned as $\mathrm{P}_{\mathrm{ox}}$, phosphorus oxide [5]. In Fig. (b), the $\mathrm{O} 1 \mathrm{~s}$ spectrum of bare $\mathrm{BP}$ has no distinct feature. But after thermal annealing, O 1s core-level spectrum in red shows two distinct components at $534.40 \mathrm{eV}$ (blue) and $532.70 \mathrm{eV}$ (green). The peaks are assigned to bridging (O-P-O) and dangling $(\mathrm{P}=\mathrm{O})$ oxygens of $\mathrm{BP}$ oxide, respectively with their intensity ratio of 3:2 [6]. It indicates that the $\mathrm{P}_{2} \mathrm{O}_{5}$-like species are formed on the BP flake after the air thermal annealing. Indeed the $\mathrm{P}_{2} \mathrm{O}_{5}$-like layer protects top $\mathrm{BP}$ layer in air conditions and realizes BP-based fieldeffect transistors [7].

\section{Conclusion}

In this work, we suggested a very simple method of fabrication of a thin and stable BP layer. By thermal annealing at $250{ }^{\circ} \mathrm{C}$ in ambient condition, we obtained the protective layer, which is composed of the phosphorus oxide, $\mathrm{P}_{2} \mathrm{O}_{5}$ on top of the BP layer. This result provides an effective and convenient strategy to fabricate high-quality stable BP-based electronics and optoelectronic devices.

\section{References}

[1] K. S. Novoselov1, et al., Science 306, 666 (2004).

[2] R. Sakamoto, et al., Nat. Commun. 6, 6713 (2015).

[3] A. Favron, et al., Nat. Mater. 14, 826 (2015)

[4] J. O Island, et al., 2D mater. 2, 011002 (2015)

[5] P. M. A. Sherwood, Surf. Sci. Spectra 9, 62 (2002).

[6] H. Kwon, et al., ACS Nano 10, 8723 (2016)

[7] S. Yang, et al., Appl. Surf. Sci. 457, 773 (2018). 